\title{
A Soft Computing Technique for Improving the Fidelity of Thumbprints Based Identification Systems
}

\author{
Kamta Nath Mishra ${ }^{1}$ \\ Department of Computer Science \& Engg., B. I. T. Mesra, Ranchi (Allahabad Campus), India \\ E-mail: mishrakn@yahoo.com \\ Anupam Agrawal ${ }^{2}$ \\ Department of CSE \& IT, Indian Institute of information Technology, Allahabad, India \\ E-mail: anupam69@gmail.com
}

\begin{abstract}
With the advent of new thumbprint identification techniques, accurate personal identification is now easy and cheaper with approximately zero false acceptance rates. This paper focuses on developing an advance feature for thumbprint based identification systems with the help of soft computing and 2D transformation which makes the technique more flexible and Fidel. The thumbprint images of individuals were scanned with the help of H3 T\&A terminal for collecting self generated datasets. The thumbprints of self generated and standard datasets were trained to form a refined set which includes linear and angular displacements of thumbprint images. The new obtained features of refined datasets were stored in the database for further identification.

In the proposed technique, the minutiae coordinates and orientation angles of the thumbprint of a person to be identified are computed and merged together for comparison. The minutia coordinates and orientation angles of a person are compared with the minutiae trained set values stored in the database at different linear and angular rotations for identity verification. The proposed technique was tested on fifty persons self generated and standard datasets of FVC2002, FVC2004 and CASIA databases. In the experimentation and result analysis we observed that the proposed technique accurately identifies a person on the basis of minutiae features of a thumbprint with low FNMR (False NonMatch Rate) values.
\end{abstract}

Index Terms - Thumbprint identification, soft computing, 2D transformation, minutiae coordinates, minutiae direction, trained set and Thumbprint patterns.

\section{INTRODUCTION}

Soft Computation techniques use few uncertain aspects to produce near to certain results. Talking about human and natural traits which can be used in an identification system, uncertain disparities and partial truth comes out along with results. Continuous perusal on thumbprints images have given few more untouched regions that can help to form better and efficient thumbprint based identification systems. A thumbprint is the biometric characteristic which is defined as oval impression and it consists of arch, left-right loops, trench and whorl etc.. Different fields in soft computing have their own importance like neural network which widely exists in many identification systems. The genetic algorithm is encoded with the help of decision variables. The fuzzy logic is also an effective tool that deals with the vagueness and uncertain aspects of the system with its major Classification: Fuzzy Set Theory and Fuzzy System. The biometric system involves several human traits like face shape; square recessive; oval dominant; Hair: Curly, Wavy, Straight; Eye shape: round recessive, Almond dominant; and Earlobes shape: attached recessive, Free lobe [10]. But, unfortunately we cannot use them for identification purpose because if we form clusters or sets based on these traits then a huge set of people will fall under single category. Therefore, human traits which we use in system for verification should follow necessary criterion like A menity (every subject should have common attribute and do not change group to group or person to person), disjunction (information shared by attributes should not be same or must vary from person to person) and indelibility (do not deplete or change over time and it should be quantifiable)

In few cases like dead bodies where finger prints are not an option and even dental evidence is difficult. In this situation palatal patterns can be taken into consideration. Palatal patterns show patterns like finger prints which bring it into category of traits that can be used for human identification [33]. The iris image also shows textures and patterns that can be quantified and used in effective identification of human but with few bottlenecks: surgery can change its original pattern [3]. The different traits which are used for personal identification are presented in fig. 1.

In general sense we can say that the proposed system should be robust, secure and can be relied over long period of time with negligible false acceptance rates. The fraternal twins are easily verifiable but identical twins 
identification is a challenging task. With increasing twin ratio every year we need to upgrade existing systems [11].

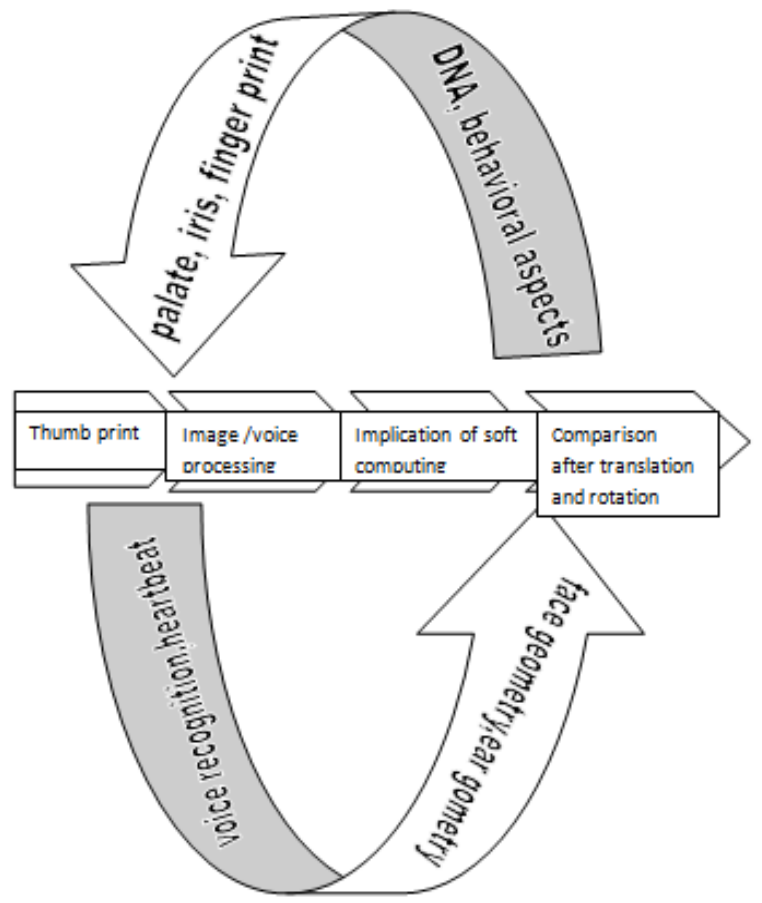

Fig.1. Identification system using different traits for personal identification.

In this paper we proposed a new refined technique that will help our existing thumbprint based identification systems to identify individual more accurately and it will also work for identical twins identification. The proposed technique of this paper is the extension of existing thumbprint based biometric identification systems which are effective in terms of space and efficient in terms of time with improved fidelity. In this paper soft computing technique along with $2 \mathrm{D}$ transformation is proposed to discover a better and flexible identification system.

In the next section (section 2) we have discussed literature review of existing systems. The section 3 describes mathematical model of the proposed system. In section 4 we have proposed new algorithms and model which uses soft computing techniques for improving the fidelity of thumbprint based systems. The section 5 analyses the performance of proposed system and the results of proposed algorithm is compared with other techniques in this section. The section 6 concludes the outcomes of this paper.

\section{LITERATURE REVIEW}

In law enforcement cases identification of suspect by some means is of national importance and latent fingerprint identification brought a solution to it but with difficulties of poor quality of ridge impressions, small finger area, and large nonlinear distortion the researches on improving technique based on finger print identification got catalyzed [15]. Finger prints are best suited trait for a real time system processing for faster computation. Therefore, Danese proposed a matching algorith $m$ based on Band-Limited Phase Only spatial Corelation (BLPOC) [7]. There were areas where you need to use other identification systems and even we can use hybrid (multi-biometric system). In one of the papers Delac et al. used face recognition technique and his experiments showed higher recognition rate with transform coefficient input to recognition algorithm as compare to the pixels of uncompressed images [8]. Zhang et al. [34] stated that if a single image based face recognition technique can produce satisfactory results then it means that the multiple images and video sequences can further improve these results. Therefore, Zhang et al. introduced probabilistic feature extraction algorithm [34].

Chen et el. studied about magnetic strip cards and found that magnetic strip are no more required if we have reliable smart card that can be used for finger print identification [6]. If we talk about Eigen values which are extracted from iris image after removing noise disturbances then we can say that the Eigen values of iris images can be used to identify individual and identical twins. Mishra K. N. et al. used smart card as storage device for storing Eigen values of iris images and these Eigen values were used for online identification of individuals and identical twins [20]. The hand geometry based identification systems showed promising results but it has a disadvantage that the hand geometry changes with time [2].

The voice based real time identification system was proposed by Phua et al. which is robust and accurate enough to identify the individuals [25]. A new biometric approach based identification technique was proposed by Ribaric et al. which has used 1820 hand images in conducting experiments and the technique showed 0.58 percent EER (equal error rate) \& 0.72 percent TER(total error rate) [26]. Jain A. K. et al. said that fingerprint textures are clear, unique and easy to process which makes fingerprint verification best for personal identification. For on line application systems Jain Anil K. et al. proposed two stage based system. In the first stage minutiae extraction from given set of image or new image took place and in second stage matching with the help of alignment-based elastic matching algorithm was used [13] [14]

In the review paper Candamo et al. described about low level processing technologies for visual surveillance which includes movement, motion tracking etc. [5]. The multimodal systems are effective and accurate over single feature based systems. Mishra K. N. et al. used Eigen iris minutiae thumb and DNA sequence features for identifying a living or a dead person. The proposed technique can identify individuals and identical twins with higher accuracy [21] [22] [23]. In this digital world we always try to build things in domain of humancomputer relation but James et al. provided a new direction by building a system that focuses upon emotions, facial expressions, gestures, postures and gaze. These features can be applied for multimodal humancomputer interaction [12]. 
Suutala et al. discussed about how a foot step can be considered as key feature to identify a person in a controlled environment. Experiments were conducted in a smart room and results showed usefulness of the system. This new extended technique has classification rate approx 95\% and rejection rate around 9\% [29]. A more reliable system proposed by Sacvic et al. which has average $\mathrm{EER}=0.0005 \%$ (equal error rate). Here, many geometric parameters of the hand like palm print; dig prints; and four finger fingers fingerprints were used where images were taken using LDA(linear discriminate analysis) [28]. Rodwlla et al. introduced the two phase head authentication technique. In the first phase registration and verification prototyping takes place and the second phase consists of experimental analysis \& authentication processes. The performance statistics of this system shows FNMR $=6 \%$ (False Non-Match Rate) and $\mathrm{FMR}=0.025 \%$ (False Match Rate) [27].

Barkhoda et al. proposed a way to deal with rotated images. The proposed steps include normalization process, extraction of blood vessels textures and thinning. The extracted features were analyzed with the help of Manhattan metric and one dimensional Discrete Fourier Transformation [4]. If we talk about accuracy and robustness of a system then a large set of sample data are to be considered for optimality of results. Aldrige et al. proposed a training data set based technique in which they used 50 images taken from a large distance like airport, space satellite, different cities and military base locations. These images were used for conducting the experiments of image processing related tasks [1]. Josphineleela et al. proposed a novel system for attendance management where they used two types of matching results for verification. In the first type the original and sample images were compared whereas in the second type the original images were compared with reconstructed scanned images [16]. Patil et al. proposed query and input minutiae images based technique for improving the performance of identification systems [24].

Kondekar et al. proposed a new technique for fingerprint identification system which is known as FHLSNN (Fuzzy Hyper Line Seg ment Neural Network). This technique uses special codes for extracting finger features [17]. Kulkarni et al. showed a new direction to fingerprint identification by introducing minutiae orientation as an extended field to minutiae coordinate in their research work. The proposed techniques showed better genuine acceptance rate (GAR) and genuine rejection rate (GRR) [18]. Han et al. proposed minutiae points and similarity between fingerprints based framework for personal identification [9]. Leung et al. used Gabor filter and neural network techniques for extracting the features of complex fingerprint images [19].

Next section states the mathematical structure of new technique proposed in this paper for improving the fidelity of identification system and few basic equations with a generalized equation which explains the relation of key elements that constructs a thumbrint image and features that helps us to determine quality image.

\section{MATHEMATICAL STRUCTURE OF PROPOSED SYSTEM}

Let us take a non empty set $\mathrm{Z}$ whose elements are $\mathrm{p}_{\mathrm{x}}$, $\mathrm{p}_{\mathrm{y}}$ and $\mathrm{p} \quad$ Here, $\mathrm{p}_{\mathrm{x}}$ represents $\mathrm{x}$ coordinate of minutiae point; $p_{y}$ represents $y$ coordinate of minutiae point; and $\mathrm{p}$ represents orientation angle. The minutia point can now be represented by Eq. (1).

$$
\text { Minutia Point }=f\left(p_{x}, p_{y}, p_{\theta}\right)
$$

The values and coordinates of $\mathrm{p}_{\mathrm{x}}, \mathrm{p}_{\mathrm{y}}$ and $\mathrm{p}$ represented by fig. 2 .

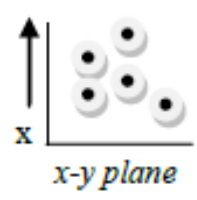

$2(a)$

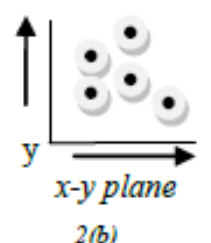

2 (b)

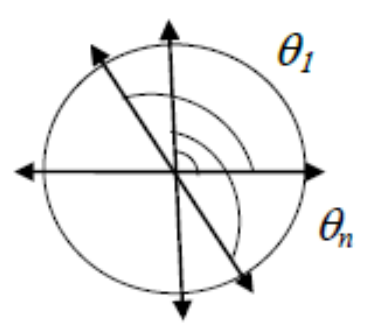

2(c)

Fig.2. Representing the orientation angles of minutiae points.

In fig. 2, x-y plane denotes the area on which we place our thumb for scanning and it can be a monitor, sensor or glass slab. To decrease system complexity we use few notifications defined over different ranges for angular displacement. Here, $\Theta$ is the range of angular displacement caused due to rotation caused while scanning thumb prints. These ranges are categorized into eight categories and every category is denoted by a numeric value from 1 to 8 . Here, 1 represents north, 2 represents north-east, 3 represents east, 4 represents south-east, 5 represents south, 6 represents south-west, 7 represents west, and 8 represents north west.

The minutiae coordinates with orientation angles of a minutia point can be defined by following function:

$$
F\left(p_{x}, p_{y}, p_{\theta}\right)=f(x, y, \theta)
$$

In Eq.(2), represents the orientation angle which may have numeric value between 1 and 8. Quantify ing images on mathematical bas is involves few computations 
which can be briefly explained in following three steps:

Step 1: Training Images.

For experimentation purpose, we take 10 thu mb print images of each individual to create a training data set on basis of following property functions:

$T\left(f_{p}\right)=\left\{f_{p}\right.$ : where, $f_{p}$ is smooth, thinned, posses clear edges, posses clear bifurcation, noise free, contain very less broken ridges, and posses sharp edges.\}

\section{Step2: Transformation of minutiae points $(T)$}

The transformation can be divided into four categories namely: translation without rotation, Rotation without translation, and translation with rotation. These categories are described in steps 2.1 and 2.2.

\section{Step 2.1 Translation without Rotation $\left(T_{t}\right)$}

In this step the minutiae points are expected with deviation from its original position without any angular rotation. This is due to improper placing of thumb on scanning area. Since, minutiae coordinates are represented in form of clusters. Therefore, the minutiae points without angular rotation can be called as a static point on plane. If there is some displacement in any minutiae point then this can cause other minutiae points to be displaced in the same proportion. This displacement can be represented by Eq.(4), Eq.(5) and Eq.(6).

$$
\begin{aligned}
& T_{x}=1 \cdot p_{x}+1 \cdot p_{x}+0 \cdot p_{y} \\
& T_{y}=0 \cdot p_{x}+1 \cdot p_{y}+1 \cdot p_{y}, \\
& T_{t}=\begin{array}{cccccc}
1 & 0 & p_{x \prime} & & p_{x} \\
0 & 1 & p_{y \prime} & \times & p_{y} \\
& 0 & 0 & 1 & & 1
\end{array}
\end{aligned}
$$

The multip lication result of Eq.(6) will be represented by Eq.(7).

$$
T_{t(x, y)}=\left\{\left(P_{x}+P_{x}\right) \times\left(P_{y}+P_{y},\right)\right\}
$$

\section{Step 2.2: Rotation without Translation}

Due to the angular movement of thumb on scanner thumbprint image shows little deviation in orientation angles. Therefore, it is very difficult for unbounded devices to get the same angled thumbprint images every time. The rotation of $\mathrm{x}$-coordinate and $\mathrm{y}$-coordinate of a minutia point can be represented by Eq.(8) and Eq.(9).

$$
\begin{aligned}
& T_{x}=p_{x} \cos \theta-p_{y} \sin \theta \\
& T_{y}=p_{x} \sin \theta+p_{y} \cos \theta \\
& T_{R}=\begin{array}{cc}
\cos \theta & -\sin \theta \\
\sin \theta & \cos \theta
\end{array} \times \quad \begin{array}{l}
p_{x} \\
p_{y}
\end{array}
\end{aligned}
$$

In Eq.(10), $T_{R}$ is a matrix which is used to perform a rotation in $x-y$ plane. Therefore, rotation of $T_{R}$ with respect to $\mathrm{x}-\mathrm{y}$ plane can be represented by Eq.(11).

$$
T_{R(x, y)}=\left[\left(p_{x} \cos \theta-p_{y} \sin \theta\right)\left(p_{x} \sin \theta+p_{y} \cos \theta\right)\right]
$$

\section{Step 2.3: Translation with Rotation}

If a thumbprint image gets linear and angular displacement while moving it from one location to another location then in this situation translation and rotation will occur simultaneously. The translation and rotation of minutiae points for a thumbprint image can mathematically represented by Eq.(12), Eq.(13) and Eq.(14).

$$
\begin{gathered}
T_{t R}=T_{(x, y)}^{\prime} T_{R} T_{(x, y)} \\
T_{x}=(x \cos \theta-y \sin \theta-d e v \times \cos \theta+d e v \times \sin \theta+d e v) \\
T_{y}=(x \sin \theta+y \cos \theta-d e v \times \sin \theta-d e v \times \cos \theta+d e v)
\end{gathered}
$$

In Eq. (12), Eq. (13) and Eq.(14) 'dev' is representing deviation from origin.

Step3: Comparison of minutiae points with the union set of minutiae points.

With the help of Eq. (7), Eq. (11) and Eq. (12) we can represent the fusion of trained input images after linear and angular translation by Eq.(15), Eq.(16), Eq.(17), and Eq.(18).

$$
\begin{gathered}
V\left(T_{t}\right)=U_{x=0, y=0}^{x=n, y=n} T_{t(x, y)} \\
V\left(T_{R}\right)=U_{x=0, y=0}^{x=n, y=n} T_{R(x, y)} \\
V\left(T_{t} T_{R}\right)=T_{t(x, y)} U T_{R(x, y)} \\
F_{V}=\left\{V\left(T_{t}\right) U V\left(T_{t^{\prime}}\right) U V\left(T_{R}\right) U V\left(T_{R^{\prime}}\right)\right. \\
\left.U V\left(T_{t R}\right) U V\left(T_{t R^{\prime}}\right)\right\}
\end{gathered}
$$

In the above equations, $\mathrm{V}\left(\mathrm{T}_{\mathrm{t}}\right)$ is representing union of minutiae points after implementing step $2.1, \mathrm{~V}\left(\mathrm{~T}_{\mathrm{R}}\right)$ is representing union of minutiae points after implementing step 2.2, $V\left(T_{t}, T_{R}\right)$ is representing the union of minutiae points after implementing step 2.3 , and $F_{v}$ is representing the union of minutiae obtained after implementing steps 2.1, 2.2 and 2.3 for the same thumbprint image.

\section{PRoposed Methodology}

It is difficult to maintain the accuracy of thumbprint scanning devices in terms of displacement and rotation. In the proposed technique we have analyzed thumbprint images with the help of 2D transformation. The propose 
technique includes the cases of rotation and translation for thumbprint images.

The proposed technique uses following approaches (discussed in sections $\mathrm{A}, \mathrm{B}$ and $\mathrm{C}$ ) for completing the tasks of processing, rotation, translation and comparison of comparis on of thumbprints.

\section{A. Proposed Algorithmfor Image Processing}

All useful images are stored in the database. We need to fetch and make them available for front end processing. These images cannot be used directly for matching results because of their improper and distorted behavior. But, with the help of smoothing, thinning and joining of thumbprint pixels we can fix most of the thumbprint images.

The gray scale images of thumbprints are processed on standard thickness $5 \mathrm{~mm}$ to $7 \mathrm{~mm}$. If once the thumbprint images start showing clear ridges then we move to next phase where training and matching is performed. For transforming and processing thumbprint images of the database we have used algorithm of fig. 3 .

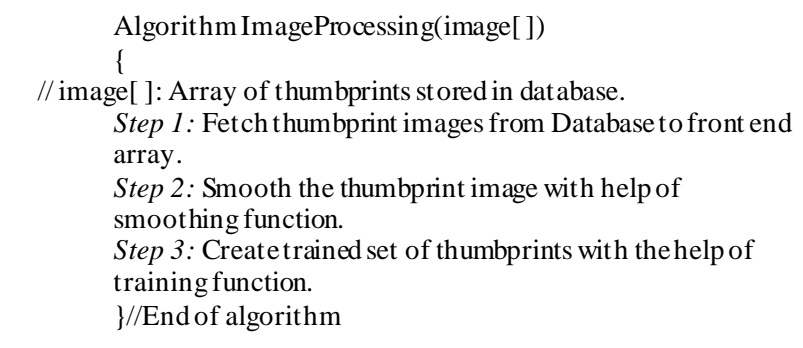

Fig.3. steps to fetch image from our thumbprint image database.

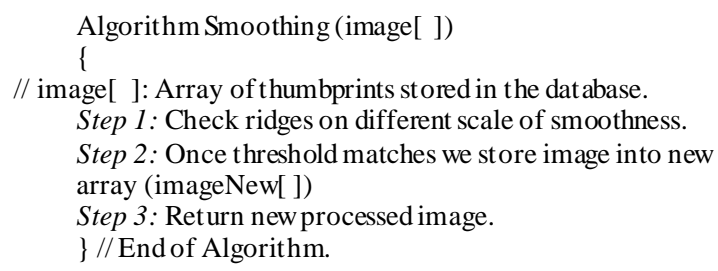

Fig.4. steps to make image smooth for further transformation.

The proposed thumbprint image s moothing algorith $\mathrm{m}$ is presented in fig. 4. The thinning task is completed with the help of sharpness variation on thumbprint images in such a way that each bifurcation should be clearly visible.

\section{B. Proposed Algorithmfor Testifying Trained Images}

Training set of thumbprint images helps us to obtain optimal images and these optimal images are used for further processing \& matching of results. In training, we test for best image on the grounds that involves clarity, broken ridges, bifurcation points, orientation angle, sharp and clear edges. The set of all these properties need to be satisfied by a thumbrint which is to be considered as an entity in a trained set.

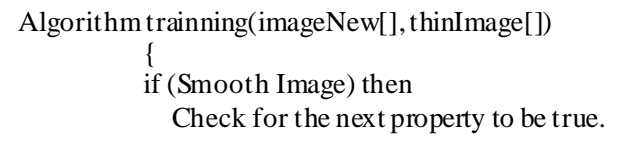

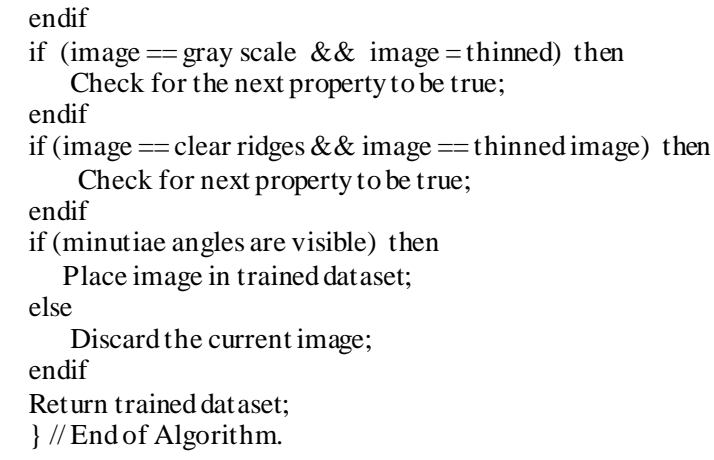

Fig.5. Steps for building and testifying trained image.

The output of fig. 5 is used for extracting minutiae coordinates and orientation angles. For calculating minutiae coordinates with orientation angles we convert the output of fig. 5 into binary image.

\section{Proposed Algorithm for Matching Results}

New obtained image is compared with our trained set images for verification. Trained set images are translated on every possible deviation that can occur during scanning. Comparison involves three basic steps namely translation without rotation, rotation without translation, translation with rotation. The description of these steps in the form of algorithm is presented in fig 6 .

Input: BinaryImage: Image in the form of coordinates and orientation angles.

NewImage: Image obtained from real time system for verification.

Output: Authentication result

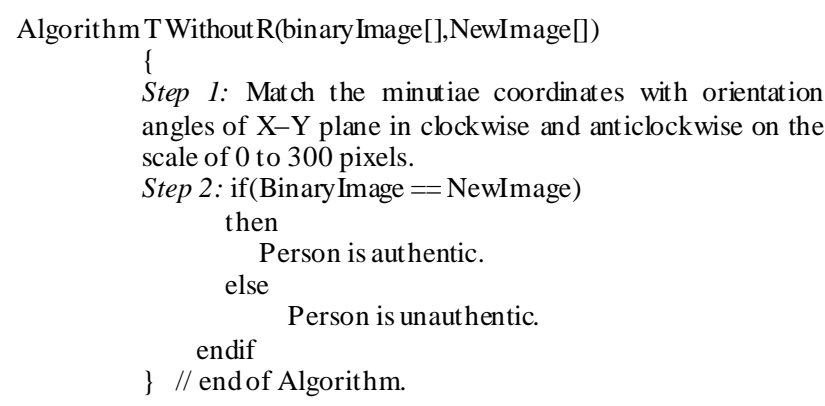

Fig.6. Steps for thumbprint image translation without rotation.

Now, a new image is compared with our trained set images for verification at every possible angular along with linear displacement using proposed algorithms of fig. 2 to fig. 6.

In the next section (experimental analysis section) we will show that how these algorithms (fig. 2, fig. 3, fig. 4, fig. 5 and fig. 6) are put together to authenticate a person after collecting his thumbprint image samples in the form of thumbprint database. Here, transforming the thumbprints on each possible deviation and angle will be taken into considerations. 


\section{EXPERIMENTAL ANALYSIS}

For result analysis we use trained set images which are obtained from original data set of thumbprint images. These thumbprints have been taken from CASIA database and some thumbprints (approximately 20 persons, 8 thumbprints of each person) were taken with the help of $\mathrm{H} 3 \mathrm{~T} \& \mathrm{~A}$ terminal. The sizes of these thumbprints vary from $2 \mathrm{~KB}$ to $3 \mathrm{~KB}$ on a screen whose lower bound and upper bound are between 0 to 300 pixels. The possible orientation angles and minutiae directions of thumbprint images are shown in fig. 6.

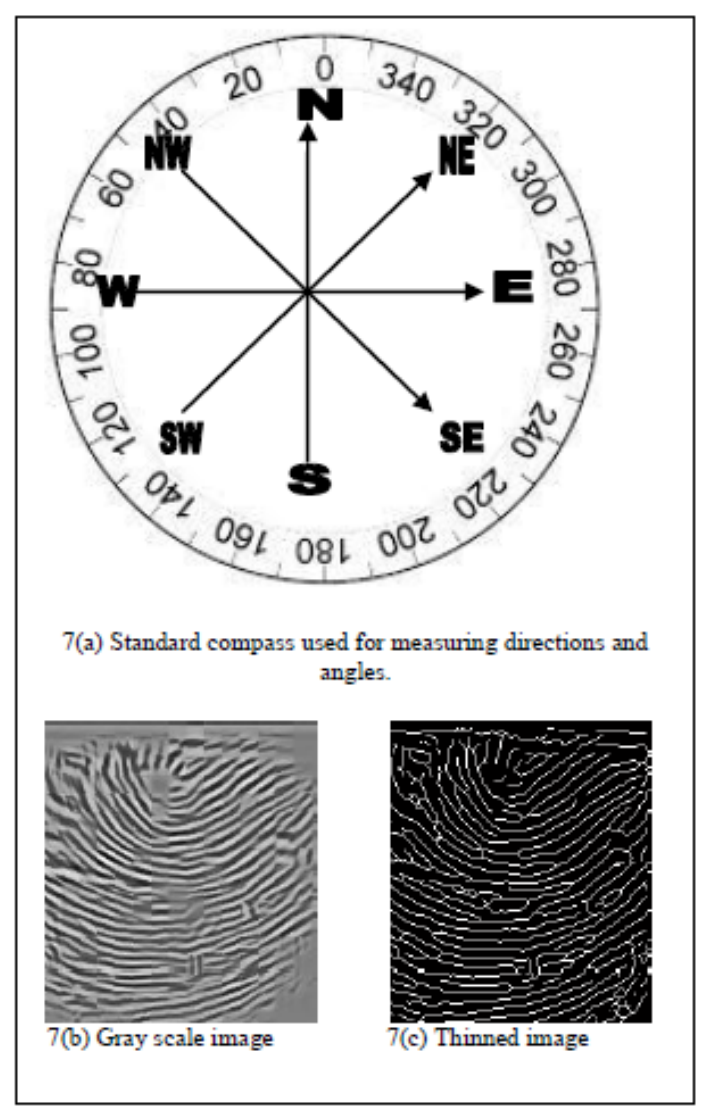

Fig.7. Standard compass used for the measurement of direction and angle with the corresponding gray and thinned images.

\section{A. Result Analysis of Translated Image}

The translated thumbprint image from a fixed point to another arbitrary point is shown in fig. 8. Their graphs are plotted on marked $x-y$ plane in such a way that the movement of every minutia coordinate can be viewed clearly with respect to its original position. The algorithms of fig. 3 to fig. 6 are used for translation.

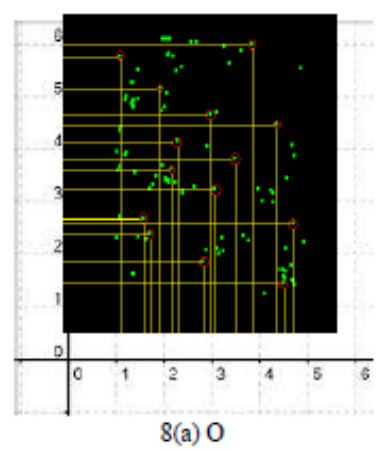

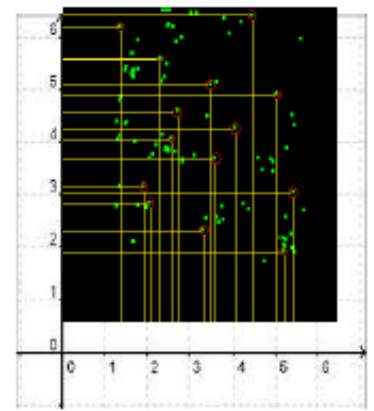

8(b) U

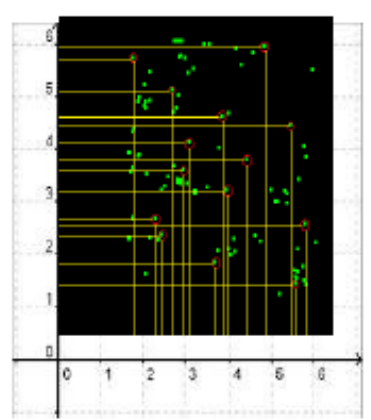

8(d) $\mathrm{R}$

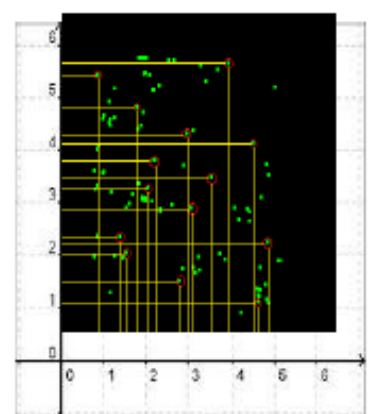

8(f) RD

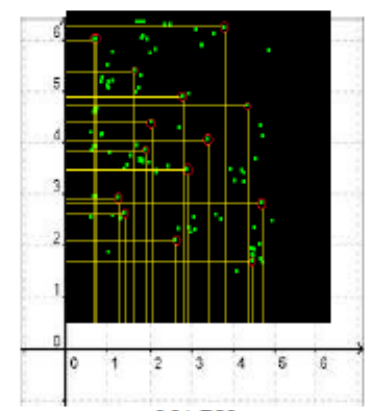

8(h) RU

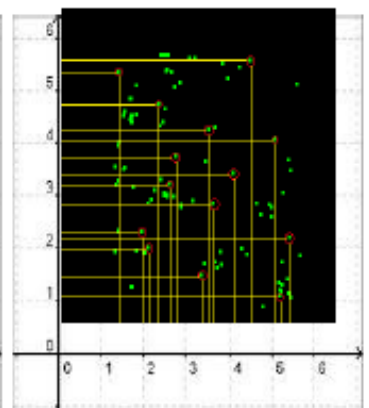

8(c) D

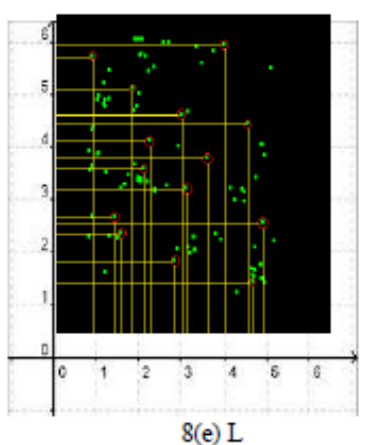

8(e) L

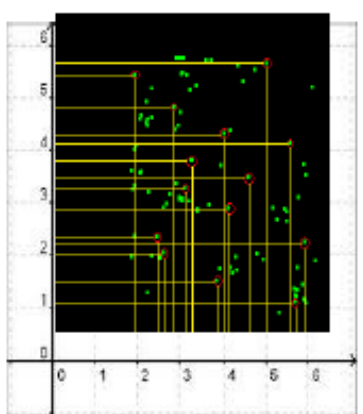

8(g) LD

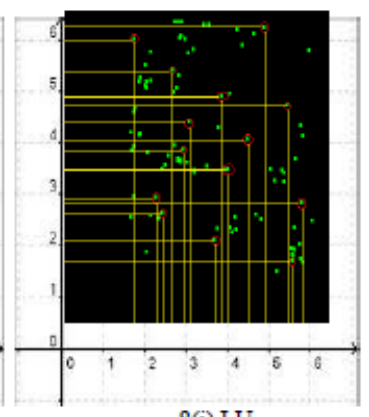

8(i) LU
Fig. 8. Minutiae points of a thumbprint image taken (before and after translation) in every possible direction. 
The fig. 8 shows the minutiae points of a thumbprint taken by considering translations and rotations. Here, ' $\mathrm{O}$ ' represents original minutiae points, ' $U$ ' represents shiftup, ' $D$ ' represents shift down, 'R' represents shift right, ' $L$ ' represents shift left, 'RU' represents right shift upwards, 'LU' represents left shift upwards, 'RD' represents right shift downwards, and 'LD' represents left shift downwards of minutiae points of a thumbprint image on $x-y$ plane. The fig. 8(a) represents the original image with no transformation where as fig. 8(b) shows shifting of minutiae coordinates on upward direction, fig. 8(c) shows shifting of minutiae coordinate towards downward direction, 8(d) shows the shifting of minutiae coordinates towards right direction, 8(e) shows the shifting of minutiae coordinates towards left direction, 8(f) shows the shifting of minutiae coordinates downwards in right direction, $8(\mathrm{~g})$ shows the shifting of minutiae coordinates downwards in left direction, 8(h) shows the shifting of minutiae coordinates upwards in right direction and fig. 8(i) shows the shifting of minutiae coordinates upwards in left direction. Here, every thumbprint image is marked with respective $\mathrm{x}$-axis and $\mathrm{y}$ axis values to see the shift on $\mathrm{x}-\mathrm{y}$ axis.

\section{B. Result Analysis of Rotated Thumbprint Images}

The original thumbprints minutiae points and the corresponding rotations from $0^{\circ}$ to $360^{\circ}$ are shown in fig 9. The minutiae coordinates can be viewed as green dots on plane black background in fig 8 . The minutiae points of fig. 9(a) are rotated and converted at different angles with the help of algorithms mentioned in figures $3,4,5$ and 6.

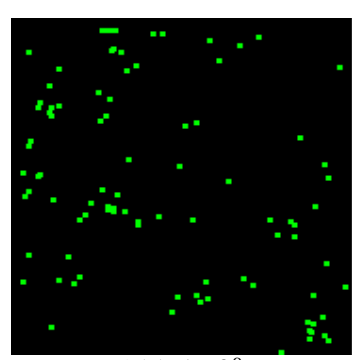

9(a) At $0^{\circ}$

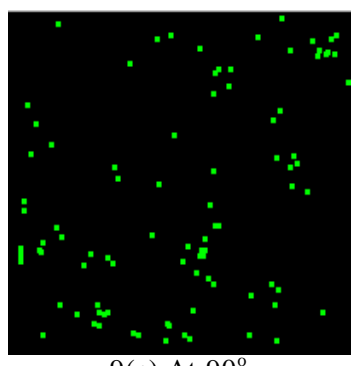

9(c) At $90^{\circ}$

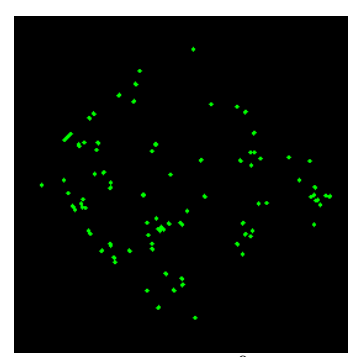

9(b) At $45^{\circ}$

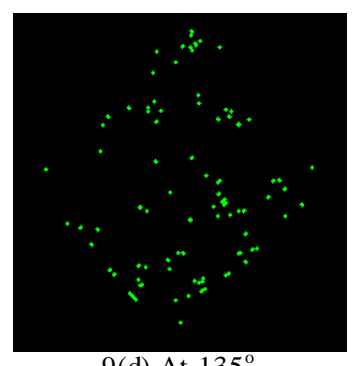

9(d) At $135^{\circ}$
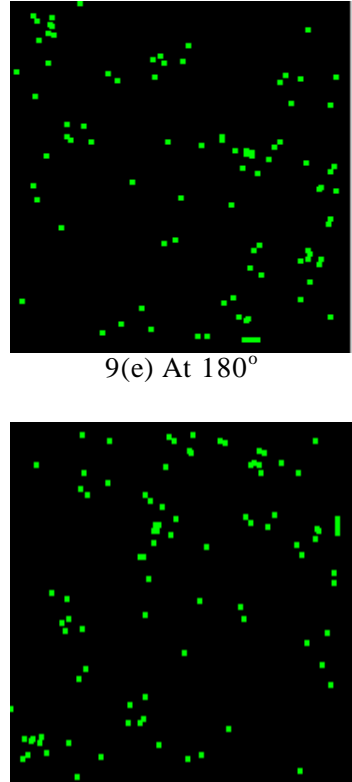

9(g) At $270^{\circ}$ 9(e) At $180^{\circ}$

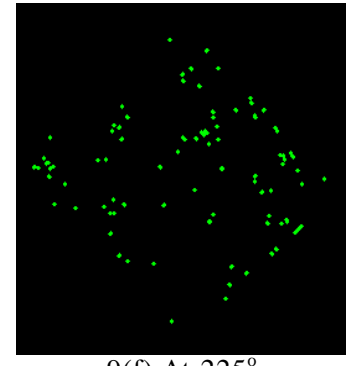

9(f) At $225^{\circ}$

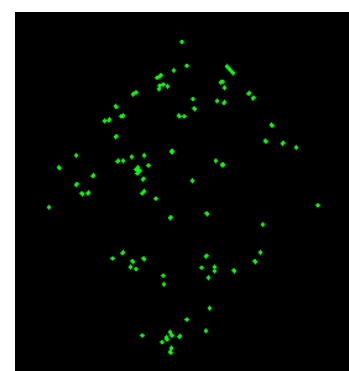

9(h) At $315^{\circ}$
Fig.9. The minutiae points of a thumbprint image taken before and after rotations in basic angles.

The fig. 9 shows the rotation of thumbprint images on every possible angle. Every real time possibility is taken into account for error correction. Here, no external modification is applied on any of the images. The fig. 9(a) is representing original image with rotation at angles $0^{0}$ and $360^{\circ}$ because both of them provide the same result. The figures 9(b), 9(c), 9(d), 9(e), 9(f), 9(g) and 9(h) are representing the rotations of fig. 9 (a) at angles $45^{\circ}, 90^{\circ}$, $135^{\circ}, 180^{\circ}, 225^{\circ}, 270^{\circ}$, and $315^{\circ}$ in clockwise directions respectively.

\section{Result Analysis at Coordinate and Angle Levels}

The minutiae coordinates with their orientation angles can be expressed in numeric terms. To understand transformation of thumbrint images it is necessary to see how minutiae coordinates and orientation angles behave with translation and rotation.

From Eq. (1) and Eq.(2) we can derive Eq.(19) which represents all the minutiae coordinates and orientation angles of a thumbprint image at all angles.

$$
\begin{aligned}
& M=\left(P_{x}, P_{y}, P_{\theta}\right) \\
& M=M_{1} U M_{2} \quad . \quad U M_{n}
\end{aligned}
$$

In Eq. (19), ' $M$ ' is representing the general structure of a minutiae point where, $\mathrm{M}_{1}=\left(\mathrm{X}_{1}, \mathrm{Y}_{1}, \theta_{1}\right), \mathrm{M}_{2}=\left(\mathrm{X}_{2}, \mathrm{Y}_{2}, \theta_{2}\right)$ and $M_{n}=\left(X_{n}, Y_{n}, \theta_{n}\right)$. 
Table 1. Deviating the minutiae points at coordinate level

\begin{tabular}{|c|c|c|c|}
\hline $\begin{array}{l}\text { Minutiae } \\
\text { Coordinates }\end{array}$ & Displacement & $\begin{array}{c}\mathrm{M}_{1} \\
(50,55)\end{array}$ & $\begin{array}{c}\mathrm{M}_{2} \\
(27,113)\end{array}$ \\
\hline $\mathrm{X}_{1}$ & +5 & 55 & 32 \\
\hline $\mathrm{Y}_{1}$ & +5 & 60 & 118 \\
\hline $\mathrm{X}_{2}$ & 5 & 55 & 32 \\
\hline $\mathrm{Y}_{2}$ & +0 & 55 & 113 \\
\hline $\mathrm{X}_{3}$ & +0 & 50 & 27 \\
\hline $\mathrm{Y}_{3}$ & +5 & 60 & 118 \\
\hline $\mathrm{X}_{4}$ & -5 & 45 & 22 \\
\hline $\mathrm{Y}_{4}$ & +0 & 55 & 113 \\
\hline $\mathrm{X}_{5}$ & +0 & 50 & 27 \\
\hline $\mathrm{Y}_{5}$ & -5 & 50 & 108 \\
\hline $\mathrm{X}_{6}$ & -5 & 45 & 22 \\
\hline $\mathrm{Y}_{6}$ & -5 & 50 & 108 \\
\hline $\mathrm{X}_{7}$ & -5 & 45 & 22 \\
\hline $\mathrm{Y}_{7}$ & 5 & 60 & 118 \\
\hline $\mathrm{X}_{8}$ & 5 & 55 & 32 \\
\hline $\mathrm{Y}_{8}$ & -5 & 50 & 108 \\
\hline
\end{tabular}

To understand the exact working of translation let us consider two minutiae points: $\mathrm{M}_{1}=(50,55,1)$ and $\mathrm{M}_{2}$ $=(27,113,6)$. The displacements of these minutiae points (without any rotation) in positive and negative directions are represented in table 1 .

Now, let us take a minutia point $M 1=(40,12,1)$ from fig. 8(a). If we rotate $\mathrm{M}_{1}$ by $45^{\circ}, 90^{\circ}, 135^{\circ}, 180^{\circ}, 225^{\circ}$, $270^{\circ}$, and $315^{\circ}$ then we get new minutiae coordinates and orientation angles namely $\mathrm{M}_{2}=(-8.4,28,2), \mathrm{M}_{3}=(-12,40$, $3), M_{4}=(-8.4,-28,4), M_{5}=(-12,-40,5), M_{6}=(8.4,-28,6)$, $\mathrm{M}_{7}=(40,-12,7)$, and $\mathrm{M}_{8}=(28,8.4,8)$. The rotation of $\mathrm{M}_{1}$ by $360^{\circ}$ will again return $\mathrm{M}_{1}$. Here, it is clear that after every rotation the rotation minutiae coordinates deviates and shifted to new coordinate but if we considered all minutiae points and analyzed their relative rotation and we observed that they have symmetry with respect to angle and displacement. All deviated minutiae coordinates starting from $\mathrm{M}_{1}$ to $\mathrm{M}_{8}$ will form same thumbprint image but at different angle and it can be seen in fig. 8 .

Therefore, the minutiae points of an image with its different rotations can be represented by Eq. (20).

$$
M_{1} \approx M_{2} \approx M_{3} \approx M_{4} \approx M_{5} \approx M_{6} \approx M_{7} \approx M_{8}
$$

For matching results and avoiding negative deviations the origin of this system $\mathrm{O}(0,0)$ is shifted to $\mathrm{O}^{\prime}(150$, 150).

\section{Result Analysis at Image Level}

Now, we know how coordinates deviate from one point to another on $x-y$ plain and how orientation angle changes its orientation. Here, we have given brief description of how results can be shown with the help of images and graphs. The peeks and groves of graphs mentioned in fig. 10 will show that how and when match percentage goes up and down with respect to the thumbprint images taken for verification purposes. The results of same and different person's thumbprints images are plotted with varying colors for better understanding. The relative results of rotation and deviation are shown with the help of graphs in fig. 11.

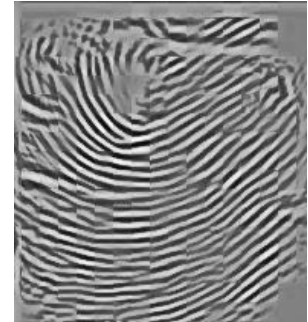

10(i) P1_IMG1

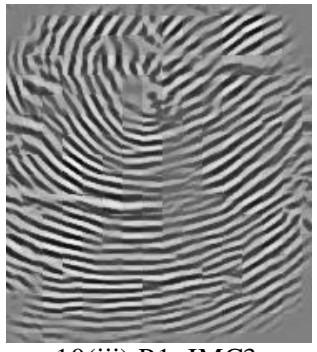

10(iii) P1_IMG3

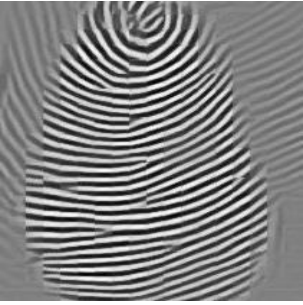

10(v) P2_IMG1

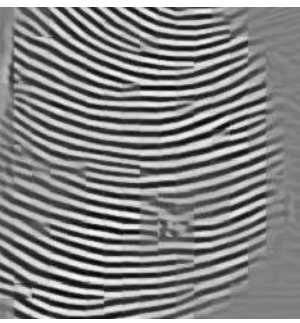

10(vii) P2_IMG3

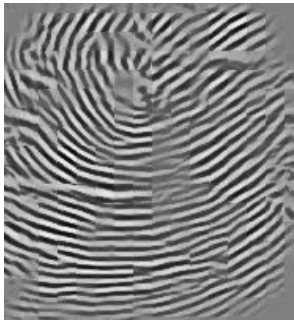

10(ii) P1_IMG2

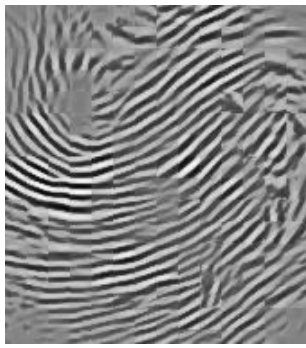

10(iv) P1_IMG4

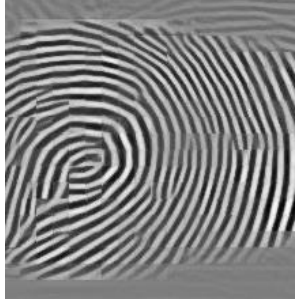

10(vi) P2_IMG2

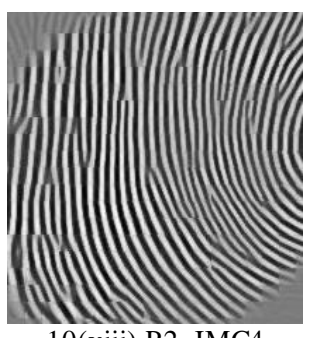

10(viii) P2 IMG4
Fig. 10. Trained set thumbprint images of two persons using H3 T \& A terminal

The thumbprints images of fig. 10 are trained with the help of soft computing techniques specified in section 4 . The thumbprint images of fig. 10 pose all necessary properties which are required to produce finest results. In real time experimentation we take a volunteer thumbprint image to form one of the sets and then we compare the volunteer image with other images at different angles and deviations.

We need to fuse data because sometimes a thumbprint may show larger values of deviations and sometimes there may exist no deviation. To avoid discrepancies and 
increase fidelity we have taken all possible conditions into consideration for translations and rotations. In the case of rotation first we collect data of images by rotating them on all possible angles then we fuse the rotated images with other remaining trained set images. If once the fusion task is over then we compare our volunteer image data with fused data to authenticate a person.

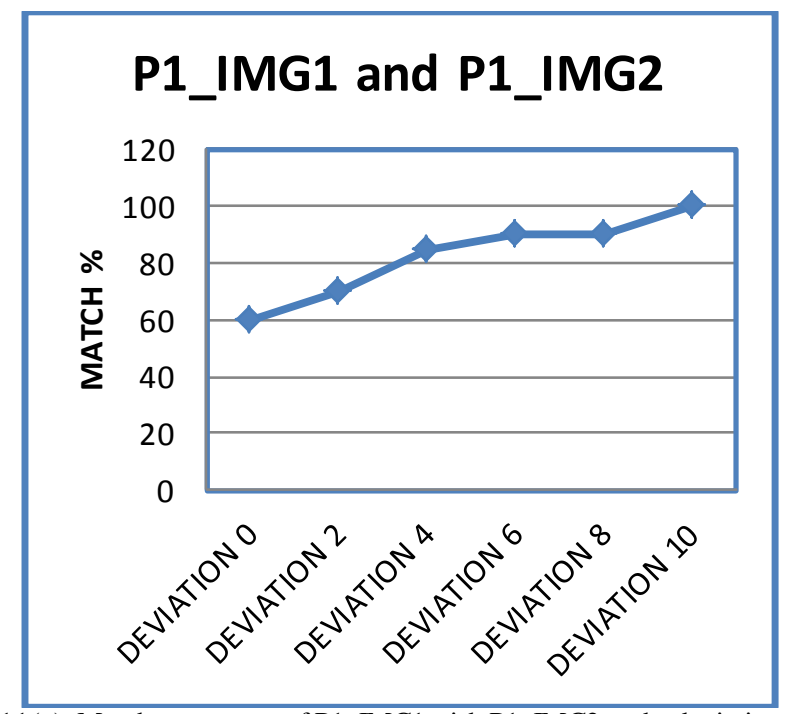

11(a). Match percentage of P1_IMG1 with P1_IMG2 at the deviation of $0,2,4,6,8$, and 10 pixels.

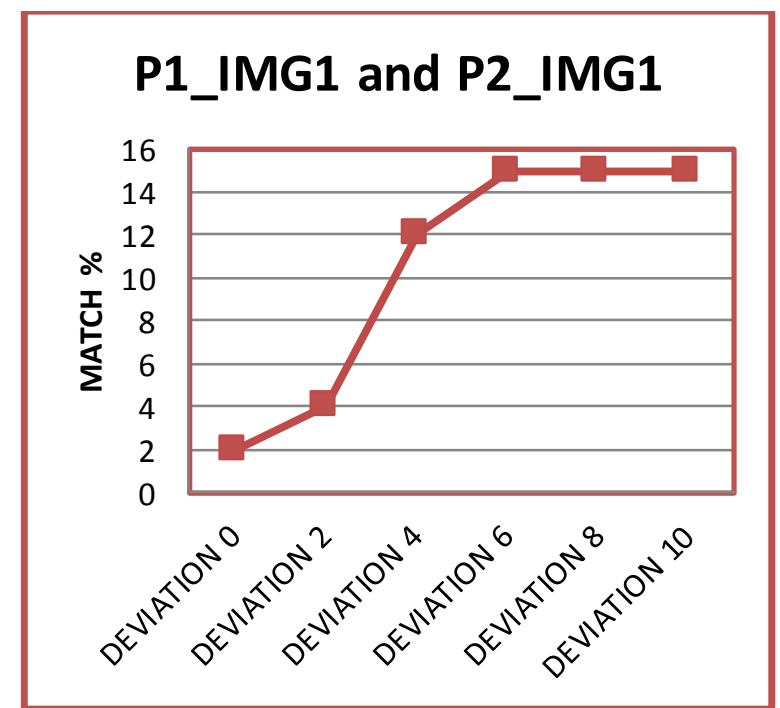

11(b). Matching the thumbprint image P1_IMG1 with P2_IMG1 at the deviation of $0,2,4,6,8$ and 10 pixels.

Fig. 11. Matching the thumbprint images of same person and different persons at different deviations

The result comparis on of thumbprint images P1_IMG1 and P1_IMG2 are shown in fig. 11. The blue line of fig. 11(a) shows that the thumbrint images have minor changes in the patterns of minutiae coordinates and orientation angles if we deviate minutiae coordinates and orientation angles by $0,2,4,6,8$ and 10 pixel values. The similar results were obtained for other self generated and standard thumbprint images of CASIA, FVC 2004 and FVC2006 data sets. After regression analysis it is observed that the match percentage for the same person's thumbprint images is finest between deviation 3 to 5 pixels.

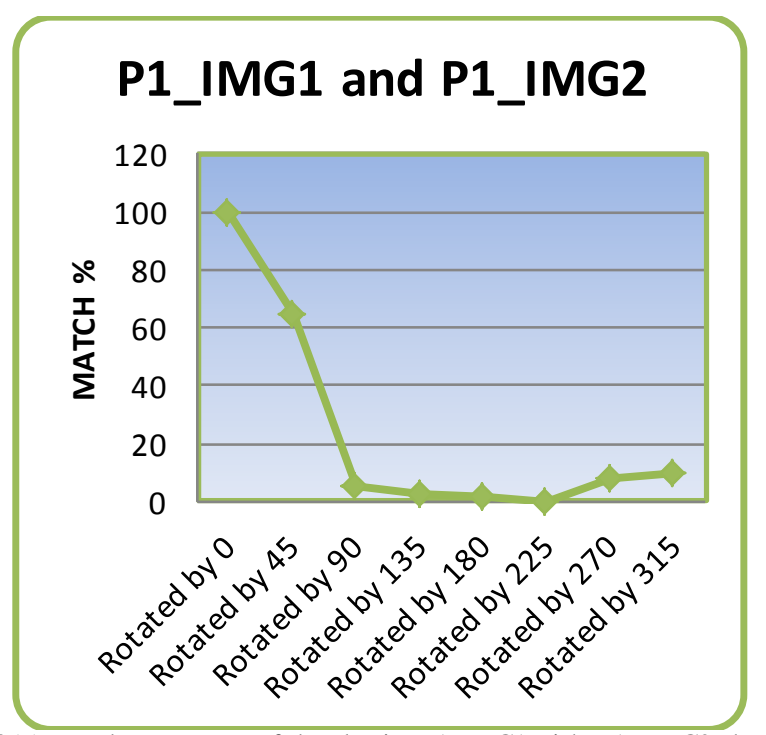

12(a) Match percentage of thumbprint P1_IMG1 with P1_IMG2 where $\mathrm{P} 1 \_\mathrm{IMG} 2$ is rotated by $0^{\circ}, 45^{\circ}, 90^{\circ}, 135^{\circ}, 180^{\circ}, 225^{\circ}, 270^{\circ}$ and $315^{\circ}$.

\section{P1_IMG1 and P2_IMG1}

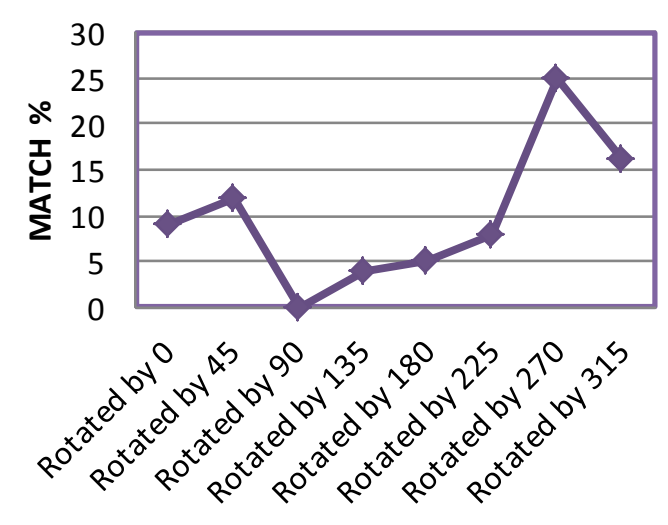

12(b) Match percentage of thumbprint image P1_IMG1 with P2_IMG2 where P2_IMG2 is rotated by $0^{\circ}, 45^{\circ}, 90^{\circ}, 135^{\circ}, 180^{\circ}, 225^{\circ}, 270^{\circ}$ and $315^{\circ}$.

Fig.12. Matching thumbprint images of same and different persons at different orientation angles.

Comparison the results of images P1_IMG1 and P2_IMG1 at deviations $0,2,4,6,8$ and 10 shown in fig. 11(b) where P1_IMG1 is the thumbprint of person 1 and P2_IMG1 is the thumbprint of person2. The results of fig. 11(b) prove that the thumbprint images P1_IMG1 and P2_IMG1 completely different from each other with respect to minutiae coord inates and orientation angles at deviations $0,2,4,6,8$ and 10 . The thumbprints of fig. 11(b) give very poor matching percentage at zero deviation. If we keep on increasing the deviation either match percentage will becomes constant or false acceptance rate will increase this is due to the fact that too much increase in deviation will allow matching two different minutiae points and it will increase the match percentage.

After every rotation performed on each thumbprint

I.J. Intelligent Systems and Applications, 2016, 7, 14-27 
image, image is displaced from its original position even its orientation suffers with little deviation. Therefore, we need to analyze the change that took place during transformation. Hence, with the help of equations Eq.(1) to Eq.(19) we need to consider its current position that is relative to original position.

The result comparisons of thumbprint images P1_IMG1 and P1_IMG2 at rotational angles $0^{\circ}, 45^{\circ}, 90^{\circ}$, $135^{\circ}, 180^{\circ}, 225^{\circ}, 270^{\circ}$ and $315^{\circ}$ using our proposed technique are shown in fig. 12(a). The results of fig. 12(a) show that the finest results are recorded around angle $0^{\circ}$ and $45^{\circ}$. We can see that the graph of fig. 12(a) is dipping after angles $45^{\circ}$ because we don't find similar coord inates due to change of orientation angles and displacements in minutiae coordinates. In order to handle the cases of rotation, the coordinates and orientation angles of minutiae points are taken at different angles. These set of values are fused together by union operation for optimizing the result set and it is used in calculating match percentage to verify identity of individuals.

The result comparison of person 1 and person2 thumbprint images P1_IMG1 and P2_IMG1 at different orientation angles are shown in fig. 12(b). While calculating the exact location of minutiae coordinates and orientation angles we have used the equations Eq.(8), Eq.(9), Eq.(10), Eq.(11) \& Eq.(12) and we have discarded the negative minutiae coordinates values which were obtained after the rotation of thumbprints. The rotation at $360^{\circ}$ angle is not mentioned in figures 12(a) and $12(\mathrm{~b})$ because at $0^{\circ}$ and $360^{\circ}$ rotations of minutiae points exactly same result is obtained.

The result comparisons of same person's thumbprint images with other persons thumbprints at different deviations and orientation angles using our proposed technique is shown in fig. 13. Now, we have compared the results of Dharmesh thumbprint image (P1_IMG1) with other thumbprint images of Dharmesh (P1_IMG3, P1_IMG4) and Mangal (P2_IMG3, P2_IMG4) at linear deviations $0,2,4,6,8$, and 10 pixels for trained set images in fig. 13(a). Every comparison of thumbprint images with P1_IMG1 is represented by different color line marker in fig. 13(a). Here, P1_IMG1 and P1_IMG3 are represented by blue line marker, P1_IMG1 and P1_IMG4 are represented by red line marker, P1_IMG1 and P2_IMG3 are represented by green line marker, P1_IMG1 and P2_IMG4 are represented by purple line marker.

Matching results of two images of fig. 13(a) and 13(b) are above $70 \%$ for the thumbrints of same person which verifies that these thumbprints belong to the same set of a person on the other hand the comparis on of P2_IMG3 and P2_IMG4 with P1_IMG1 stayed below $30 \%$ of matching which verifies that thumbprint images (P2_IMG3 and P2_IMG4) do not belong to the set of P1_IMG1. But, in some special cases few thumbprint images have different orientation angles and our technique of deviation refuse to verify those images.

Therefore, to handle these cases we have used another technique where angular and linear displacements are considered together for comparing thumbprint images.

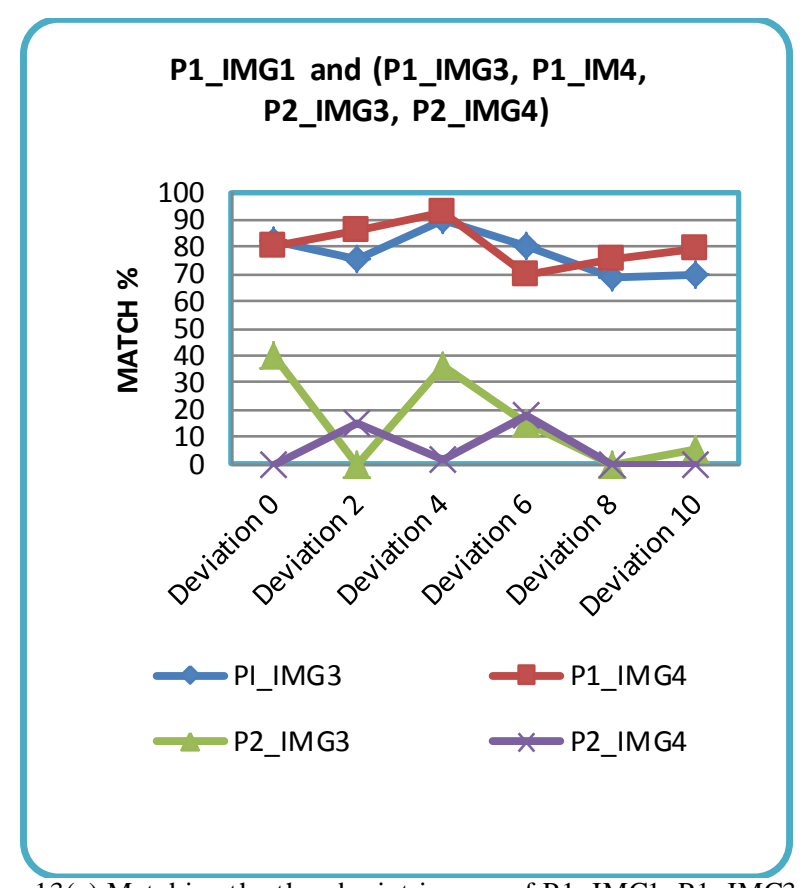

13(a) Matching the thumbprint images of P1_IMG1, P1_IMG3, P1_IMG4,P2_IMG3 and P2_IMG4 with each other at the deviations of $0,2,4,6,8$ and 10 pixels.

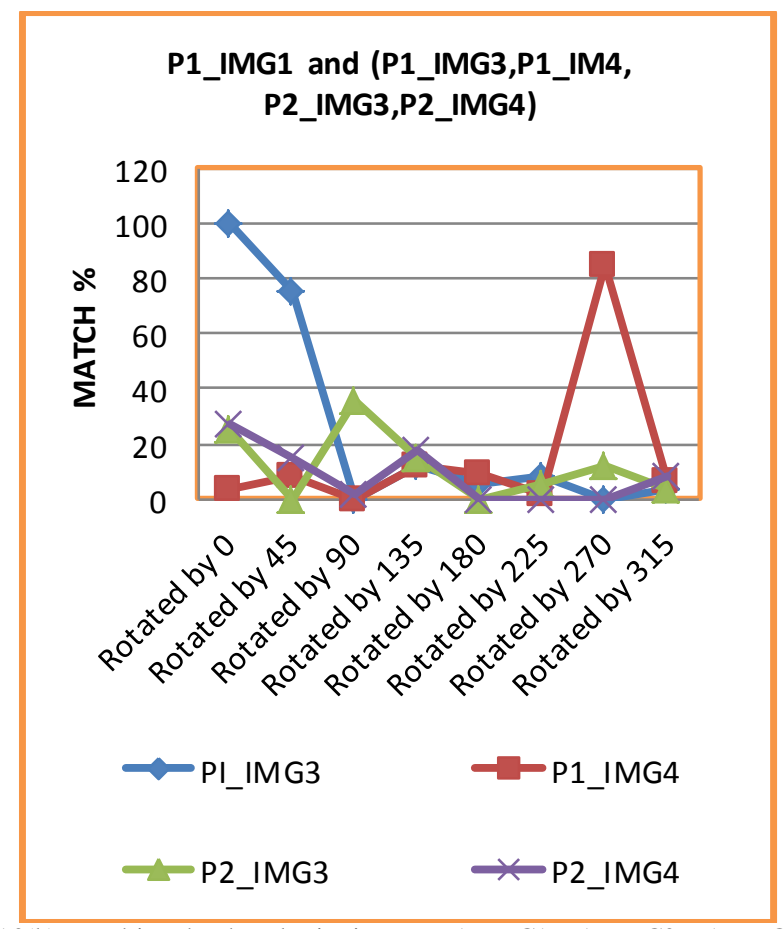

13(b) Matching the thumbprint images P1_IMG1, P1_IMG3, P1_IMG4, P2_IMG3 and P2_IMG4 with each other after rotating the images by $0^{\circ}$, $45^{\circ}, 90^{\circ}, 135^{\circ}, 180^{\circ}, 225^{\circ}, 270^{\circ}$ and $315^{\circ}$.

Fig.13. Matching the thumbprint images of same person and different persons at different linear and angular deviations.

Hence, we can now clearly identify which thumbrint images belong to the same set and which thumbprint images belong to a different set. Now, let us see what we get in relative comparison of thumbprints after including linear and angular rotations together in fig. 13(b).

In fig. 13(b) the thumbrint P1_IMG1 is compared with P1_IMG3, P1_IMG4, P2_IMG3 and P2_IMG4 after 
including linear deviations and rotating them by $0^{\circ}, 45^{\circ}$, $90^{\circ}, 135^{\circ}, 180^{\circ}, 225^{\circ}, 270^{\circ}$ and $315^{\circ}$. The comparis on of thumbprint images with P1_IMG1 after considering deviations rotations are represented by different color line markers e.g., in fig. 13(b), the comparison of P1_IMG1 with P1_IMG3 at different linear and angular rotations are represented by blue line marker, the comparison of P1_IMG1 with P1_IMG4 at different deviations (linear and angular) are represented by red line marker, Similarly, the comparis on of P1_IMG1 with P2_IMG3 and P2_IMG4 at different angular and linear deviations are represented by green and purple line markers.

\section{E. Result Analysis with Standard Databases (FVC2002, 2004 and CASIA)}

The FVC2002, FVC2004 and CASIA databases are standard database which are used for analyzing the performance of biometric systems. The size of all individual thumbprints for FVC2002 and FVC2004 database is ranging from $105 \mathrm{~KB}$ to $115 \mathrm{~KB}$ whereas the size of a thumbprint image for CASIA database is $115 \mathrm{~KB}$ to $120 \mathrm{~KB}$ approximately. We compared 50 people's thumbprint images of CASIA, FVC2002, FVC2004 databases and observed that the proposed technique successfully identifies individuals. Although, the thumbprint images taken from FVC2002 and FVC2004 are shifted left and right at different angles of orientation but still the proposed technique is able to clearly differentiate the thumbprints of FVC2002, FVC2004 and CASIA data sets.

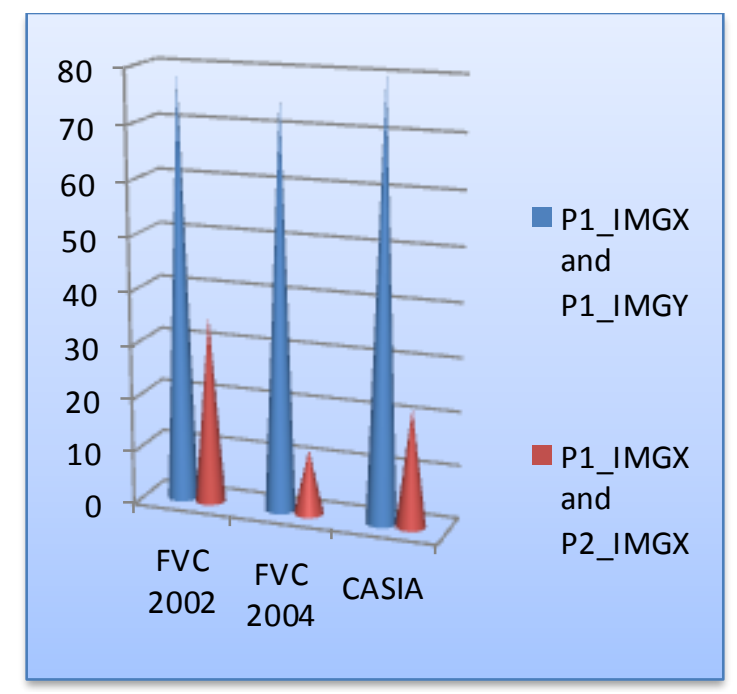

Fig. 14. Matching the results of FVC and CASIA dataset thumbprint images at different linear deviations (1 pixel to 10 pixels).

In the graphs of fig. 14 and fig. 15, the comparis on of results of eleven thumbprint images taken from FVC2002(P11_IMG00, P11_IMG11, P11_IMG12, P11_IMG13, P11_IMG14, P11_IMG15, P12_IMG11, P12_IMG12, P12_IMG13, P12_IMG14, P12_IMG15) database, eleven thumbprint images taken from FVC2004 (P21_IMG00, P21_IMG21, P21_IMG22, P21_IMG23, P21_IMG24, P21_IMG25, P22_IMG21, P22_IMG22,
P22_IMG23, P22_IMG24, P22_IMG25) database and eleven thumbprint images taken from CASIA database(P31_IMG00, P31_IMG31, P31_IMG32, P31_IMG33, P31_IMG34, P31_IMG35, P32_IMG31, P32_IMG32, P32_IMG33, P32_IMG34, P32_IMG35) are shown at different linear and angular deviations. In the experimentation it is observed that at the linear deviations 4 and 6 the best comparison results were obtained. It is further observed in experiments that the rotation of thumbprints at different angles between 0 and 90 degrees have the minimum effect on matching percentage for the proposed technique.

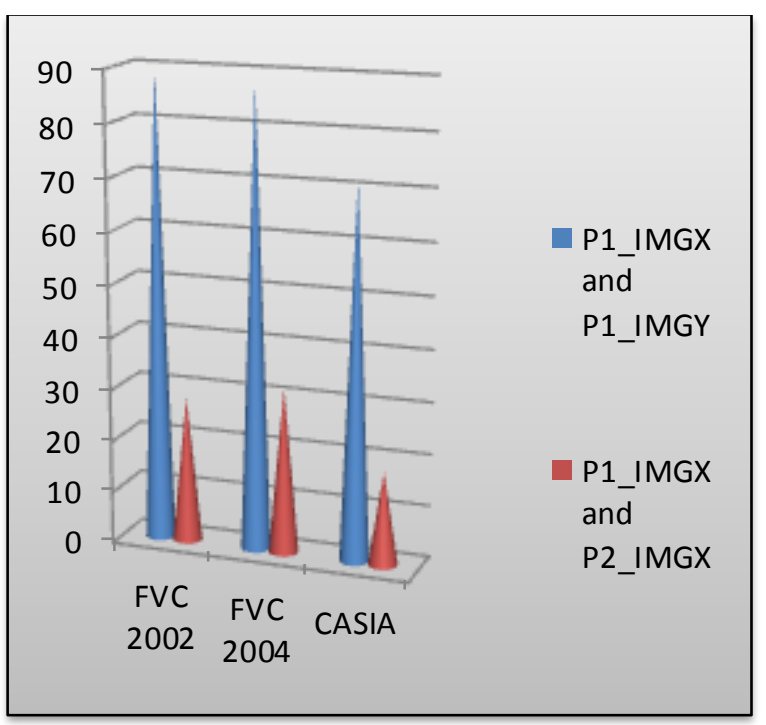

Fig. 15. Matching the results of FVC and CASIA dataset thumbprint images at different angular deviations.

\section{F. Comparing the Results of Proposed Technique with Standard Techniques}

The technique proposed in this paper is tested on thumbprints of fifty persons self generated thumbprints and fifty individuals thumbprint images of FVC and CASIA database of thumbprint images. The technique defined and proposed in this paper works efficiently on the standard databases (FVC2002, FVC2004, CASIA) and self generated thumbprint images which are taken with full attention and have minimal shift towards right, left, up \& down on device with little angular displacements. The minutiae points are the bifurcation points or breakage points as discussed in the starting of this paper. Most of the techniques discussed in table 2 use minutiae points as key characteristic for comparison. The minutiae coordinates include $\mathrm{x}$-coordinate, $\mathrm{y}$ coordinate and in few techniques orientation angle and directions are also used.

If thumbprint images have considerable amount of deviation and orientation angles of thumbprints are differing from each other then in this case standard techniques will fail to match same person's thumbprints. In a real time system we are forced to maintain minimum time complexity but the exiting standard system will either reject few scan or ask for new thumbprint image. The thumbprint images with higher values of deviations 
will increase time complexity in image processing. To avoid unnecessary rejection and to decrease the time complexity which is paramount in real time system of identification we have proposed a new technique in this research work which is considering linear and angular displacements.

Table 2. Results of existing methods and proposed method

\begin{tabular}{|l|l|l|}
\cline { 2 - 3 } \multicolumn{1}{c|}{} & $\begin{array}{l}\text { Algorithm } \\
\text { (Matcher Name) }\end{array}$ & Matching Results \\
\hline 1. & Jain [13] (Minutiae) & FAR: 2 - 6\% higher \\
3. & Han [6] (Minutiae ) & EER: 1 - 2\% higher \\
4. & Srihari [31] (NFIS) & EER: 6.17\% \\
5. & Sun [30] (VeriFinger ) & EER: 6.79\% \\
6. & Mishra [22] (IT IS) & EER: 5\% \\
7. & Mishra [20] (MFBFTI) & EER: 2\% approx. \\
8. & Proposed & EER: 1.5\% to 2\% approx. \\
\hline
\end{tabular}

The low FNMR increases the efficiency of any identification system. In the proposed technique we focused on decreasing FNMR (False Non-Match Rate) which is around $1.5 \%$ to $2 \%$ approximately. It is observed that no other technique was able to achieve such low FNMR. The proposed identification technique does not discard any thumbprint image unless it is of very poor quality. The FAR (False Acceptance Rate) of our proposed technique is around $2 \%$ to $5 \%$ approximately. The proposed technique reduces the manual task of thumbprint based image processing systems. The comparison between proposed technique and other exiting standard techniques of table 2 shows that the proposed systems is Fidel and it is best suited for dynamic and real time identification.

\section{CONCLUSIONS}

In this paper we proposed a soft computing technique which performs image processing tasks with the help of 2D graphical transformation and it increases the fidelity of identification system. It is a proven fact that match percentage of two thumbprint images will increase if we compare them on different linear and angular deviations. The deviations and rotations are two different techniques discussed in this paper which can be used to increase the efficiency and fidelity of identification systems. We have combined the deviations and rotations together to form a hybrid approach that will take care of deviations and rotations together at the same point of time.

Our proposed method successfully works for existing standard databases (FVC2002, FVC2004 and CASIA) and self generated database with a match threshold of $80 \%$ and above for authenticating the thumbprints of on purpose. The efficiency of proposed system with respect to time complexity is improved because of very low
FNMR. This technique is well suited for dynamic and real-time identification systems.

This research work may be used to derive socio behavioral features from thumbprints which can tell us about behavioral entities of the persons. Fuzzy set and neural network can also help to understand vagueness and density of systems behaviors. The soft and hard computing techniques together are used in this paper to generate training data sets and it is refining the performance of existing techniques. This paper proposes a technique which involves the union of new thumbprint images of different linear and angular deviations.

\section{REFERENCES}

[1] Aldridge S, "Image Sets For The Training Of Image Processing", GECCO '10 Proceedings of the 12th annual conference companion on Genetic and evolutionary computation, p. 2039-2042, 2010.

[2] Amayeh G, Bebis G, Erol A, and Mircea Nicolescu "Hand-based verification and identification using palmfinger segmentation and fusion", Computer Vision and Image Understanding, Vol. 113, No. 4, 477-501, 2009.

[3] Arora S.S, Vatsa M, Singh R, and Jain A, "Iris recognition under alcohol influence" Biometric(ICB), 5th IEEE IAPR International Conference on Biometrics Compendium, p. 336-341, 2012.

[4] Barkhoda W, Tab F. A, and Amiri M. D," Rotation Invariant Retina Identification Based On The Sketch Of Vessels Using Angular Partitioning", IEEE International Multi-conference on Computer Science and Information Technology, p. 3-6, 2009.

[5] Candamo J, Shreve M, Dmitry B. Goldgof Deborah B. Sapper, and Kasturi R, "Understanding Transit Scenes: A Survey on Human Behavior-Recognition Algorithms", IEEE Transactions on Intelligent Transportation Systems, Vol. 11, No. 1, 2010.

[6] Chain T. P, Yau Wei-Yun, and Jiang X, "Token-Based Fingerprint Authentication", Recent Patents on Computer Science, Vol. 2, p. 50-58, 2009.

[7] Danese, M. Giachero, Leporati F., and Nazzicari N., "An embedded multi-core biometric identification system $3 \mathrm{G}$ ". $13^{\mathrm{TH}}$ European Conference on Digital System Design: Architectures, Methods and Tools (DSD), p. $779-784$, 2010.

[8] Delac K., Grgic M., and Grgic S., "Face recognition in JPEG and JPEG2000 compressed domain", Image and Vision Computing ,Vol. 27, No. 8, p. 1108-1120, 2009.

[9] Han Y, Ryu C, Moon J, Kim H, and Choi H., "A study on evaluating the uniqueness of fingerprints using statistical analysis", Information Security and Cryptology ICISC04, p. $467-477,2004$.

[10] Human Traits: Autosomal, http://faculty.southwest.tn.edu/jiwilliams/Human_Traits.h tm, last seen $1 / 17 / 2014$

[11] Indi S.T., and Raut D.S., "Biometric Feature Based Person Unique Identification System", International Journal of Computer Application, Vol. 51, No. 13, p. 7-12, 2012.

[12] Jaimes A, and Sebe N., "Multimodal human-computer interaction: A survey", Computer Vision and Image Understanding, Vol. 108, No. 2, p. 116-134, 2007.

[13] Jain A. K, Lin Hong, and Ruud Bolle, "On-Line Fingerprint Verification", Proceedings of $13^{\text {th }}$ International Conference on Pattern Recognition, Vol. 3, p. $596-600,1996$. 
[14] Jain A. K., Prabhakar S., and Pankanti S., "On the Similarity of identical twin fingerprint", Pattern Recognition, Vol. 35, p. 2653-2663, 2002.

[15] Jain A. K., Feng J., "Latent Fingerprint Matching" IEEE Computer Society, Vol. 33, No. 1, p. 88-100, 2011.

[16] Josphineleela R., and Ramakrishnan M., "An Efficient Automatic Attendance System Using Fingerprint Reconstruction Technique", International Journal of Computer Science and Information Security, Vol. 10, No. 3, p. 1-6, 2012.

[17] Kondekar M.H, Kulkarni U.V., and Krishna Kanth B.B.M., "Extended Fuzzy Hyperline Segment Neural Network for Fingerprint Recognition", International Journal of Biometrics and Bioinformatics, Vol. 5, No. 3, p 162-171, 2011.

[18] Kulkarni J.V., Patil B.D., and Holambe R. S., "Orientation Feature for fingerprint matching “, Pattern Recognition, Vol. 39, p. 1551-1554, 2006.

[19] Leung M., Engeler W., and Frank P. , "Fingerprint Image Processing Using Neural Network", IEEE TENCON conference on Computer and Comm. System, p. 582-586, 1990.

[20] Mishra K. N., Srivastava P. C., Agrawal Anupam, Tripathi V., and Gupta V. "An Efficient Eigen Values Based Technique For Online Iris Image Compression and Identification" International Journal of Information Acquisition, Vol. 8, No. 2, 2011.

[21] Mishra K. N., Srivastava P. C., and Agrawal Anupam, “A Framework Towards Using Eigen Iris, Minutia Thumb and DNA Sequence Features For Personal Identification", International Journal of Information Acquisition, Vol. 8, No. 3, p. 197-225, 2011.

[22] Mishra K. N., Srivastava P. C., Agrawal Anupam, Tripathi V., and Garg R., "Minutiae Distances and Orientation Fields Based Thumbprint Identification of Identical Twins", International Journal of Image Graphics and Signal Processing, Vol. 5, No. 3, p. 51-59, 2013.

[23] Mishra K. N., Srivastava P. C., Agrawal Anupam, Garg R. and Singh A., "Minutiae Fusion Based Framework for Thumbprint Identification of Identical Twins", International Journal of Intelligent system and application, Vol. Vol. 5, No. 2, p. 84-101, 2013.

[24] Patil D.S., and Patil S.A., "Fingerprint recognition using minutia matching", World Journal of Science and Technology, Vol. 2, No. 4, p. 178-181, 2012.

[25] Phua K., Chen J., Dat T. H., "Heart sound as a biometric", Pattern Recognition, Vol. 41, No. 3, p. 906-919, 2008.

[26] Ribaric S., Fratric I., "A Biometric Identification System Based on Eigenpalm and Eigenfinger Features", IEEE Transactions on Pattern Analysis and Machine Intelligence, Vol. 27, No. 11, 2005.

[27] Rodwella P. M., Furnella S.M., and Reynold P. L., “A non-intrusive biometric authentication mechanism utilizing phy siological characteristics of the human head", Computers \& Security, Vol. 26, No. 7-8, p. 468-478, 2007.

[28] Savǐc T., and Paveši'c N., "Personal recognition based on an image of the palmar surface of the hand", Pattern Recognition, Vol. 40, No. 11, p. 3152-3163, 2007.

[29] Suutala J., and Ning J.R., "Methods for person identification on a pressure-sensitive floor: Experiments with multiple classifiers and reject option", Information Fusion, Vol. 9, No. 1, p. 21-40, 2008.

[30] Sun Zhenan, Paulino A., Feng J., and Chai Z., "A Study of Multi-biometric Traits of Identical Twins", Proc of SPIE, Biometric Technology for Human Identification, Vol. VII, p. $7667-76727,2010$.
[31] Srihari Sargur N., Srinivasan H., and Fang G., "Discriminability of Fingerprints of Twins", Journal of Forensic Identification, Vol. 58, No. 1, p. 109-127, 2008.

[32] Tao X., Chen X., Yang X., and Tian J., "Fingerprint Identification with Identical Twin Fingerprints", PLoS $\begin{array}{lllll}\text { One, } & \text { Vol. } & \text { 7, } & \text { No. } 35704,\end{array}$ (doi:10.1371/journal.pone.0035704), 2012.

[33] Thomas C. J., Van Wyk CWJ, "The palatal rugae in an identification”, Forensic Odontostomatol, Vol. 6, No. 1, p. 21-7, 1988.

[34] Zhang Y, and Martınez A. M., “A weighted probabilistic approach to face recognition from multiple images and video sequences", Image and Vision Computing, Vol. 24, No. 6, p. 626-638, 2006.

\section{Authors' Profiles}

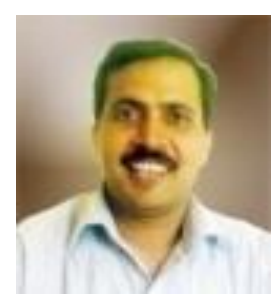

Kamta Nath Mishra was born on August 15, 1973 in Kushinagar district of Uttar Pradesh, INDIA. He received his Bachelor of Science (B.Sc., Maths) degree from University of Gorakhpur, Gorakhpur, INDIA, in 1991, Master of Computer Application (MCA) degree from Madan Mohan Malviya University of Technology, Gorakhpur, U. P., INDIA in 1996, M.Tech. (Software Systems) degree from Birla Institute of Technology and Science (BITS) Pilani, INDIA in 2003, and Ph.D. in Engg. from CSE department of B.I.T. Mesra, in May 2015. Currently, he is working as an Assistant Professor (senior grade) at Dept. of CS\&E, B.I.T. Mesra, Ranchi, INDIA (Allahabad Campus) since August 2009.

Dr. Mishra has worked as a faculty member in the department of Computer Science, University of Sebha, Sebha, LIBYA, from October 2006 to July 2009. He was a senior lecturer at B.I.T. Mesra, Ranchi, INDIA (Noida Campus) from July 2004 to September 2006. Dr. Mishra has worked as a senior project engineer (Sr. Lecturer grade) from September 2003 to June 2004 and project engineer (Lecturer grade) from September 2000 to August 2003, in Centre for Development of Advanced Computing (Ministry of Communication \& IT, Govt. of India) Noida, Uttar Pradesh.

Before joining CDAC, Dr. Mishra worked as a lecturer in CS\&E department at Krishna Institute of Engineering \& Technology (KIET), Ghaziabad, INDIA, from July 1998 to August 2000. His research interest includes Biometric Systems, Image Processing, and Analysis of Algorithms. Dr. Mishra is a professional member of IEEE Biometric Society, USA, and he has published more than fifteen research papers in journals and conferences of international repute.

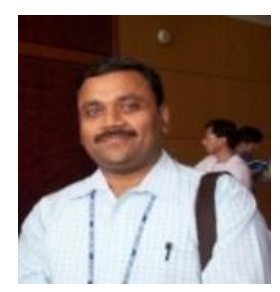

Anupam Agrawal is presently working as Professor of Computer Science and Information Technology at Indian Institute of Information Technology Allahabad (IIIT-A). Before joining IIIT$A$ in the year 2000, he was working as scientist 'D' at DEAL, DRDO, Govt. of India, Dehradun. He received his M.Tech. degree in Computer Science and Engineering from Indian Institute of Technology Madras, Chennai and Ph.D. degree in Information Technology from Indian Institute of Information Technology Allahabad (in association with Indian Institute of Technology, Roorkee). 
He was a postdoctoral researcher at the Department of Computer Science \& Technology, University of Bedfordshire (UK) during which he had contributed significantly in two major European projects. His research interests include Computer Vision, Image Processing, Visual Computing, SoftComputing and Human-Computer Interaction. He has more than 100 publications related to these areas in various international \& national journals and conference proceedings, and has co-authored four book. He is on the review board for various international journals including IEEE, Springer, MDPI, Taylor \& Francis and Elsevier. He is currently serving as a Principal Investigator of an international (Indo-UK) Project. He is a member of ACM (USA), senior member of IEEE (USA) and a fellow of IETE (India).

How to cite this paper: Kamta Nath Mishra, Anupam Agrawal, "A Soft Computing Technique for Improving the Fidelity of Thumbprints Based Identification Systems", International Journal of Intelligent Systems and Applications (IJISA), Vol.8, No.7, pp.14-27, 2016. DOI: 10.5815/ijisa.2016.07.02 